\title{
Brain State Intelligence and Cognitive Health Through EEG Date Modeling
}

\author{
Hong Lin, University of Houston-Downtown, USA \\ iD https://orcid.org/0000-0003-1827-5507 \\ Jonathan Garza, University of Houston-Downtown, USA \\ Gregor Schreiber, Chevron-Phillips Chemical Company, USA \\ iD https://orcid.org/0000-0001-8701-2992 \\ Minghao Yang, University of Houston-Downtown, USA \\ Yunwei Cui, Towson University, USA
}

\begin{abstract}
Electroencephalographic data modeling is widely used in developing applications in the areas of healthcare, as well as brain-computer interface. One particular study is to use meditation research to reach out to the high-end applications of EEG data analysis in understanding human brain states and assisting in promoting human healthcare. The analysis of these states could be the initial step in a process to first predict and later allow individuals to control these states. To this end, the authors begin to build a system for dynamic brain state analysis using EEG data. The system allows users to transit EEG data to an online database through mobile devices, interact with the web server through web interface, and get feedback from EEG data analysis programs on real-time bases. The models perform self-adjusting based on the data sets available in the database. Experimental results obtained from various machine-learning algorithms indicate great potential in recognizing user's brain state with high accuracy. This method will be useful in quick-prototyping onsite brain states feedback systems.
\end{abstract}

\section{KEYWORDS}

Brain-Computer Interface, Data Analysis, Dynamic Self-Adjusting Modeling, Electroencephalographic Analysis, Web Interface

\section{INTRODUCTION}

Using electroencephalographic (EEG) data, cognitive psychologists can visualize and observe correlations between different active brain states. It is desirable to create an application that takes EEG data and exposes it to various analytical techniques so the resultant brain states can be studied and predicted. We present explanations of the design and implementation offered herein.

An EEG device can record the electric signals from a human scalp. EEG devices used to be only available in professional health care institutions for clinic use. Last decade witnessed the development of cheap EEG devices, for example, EPOC from Emotiv (http://www.emotiv.com) and NeuroSky (http://www.neurosky.com/), and increasing interest in EEG based brain-computer interfaces (BCI). EEG signals characterize the result of the neuron activities inside of a human brain. Naturally, they are used to study and understand human brain activities. In particular, EEG signals indicate that 
neural patterns of meanings in each brain occur in trajectories of discrete steps, whist the amplitude modulation in EEG wave is the mode of expressing meanings (Freeman, 2000). Zhou et al (2008) have proposed some novel features for EEG signals to be used in brain-computer interface (BCI) system to classify left and right hand motor imagery. The experimental results have shown that based on the proposed features, the classifiers using linear discriminant analysis, support vector machines and neural network achieve better classification performance than the BCI-competition 2003 winner on the same data set in terms of the criteria of either mutual information or misclassification rate. Dressler et al studied the anaesthetics on the brain and the level of sedation (Dressler et al, 2004). Lin et al (2010) studied the change of human emotion during music listening through EEG signals.

The vast implications of using EEG data to analyze brain states include designing brain-computer interfaces $(\mathrm{BCI})$ where users can operate on a machine via brain activities, and using brain state models in healthcare related activities. As an example, we present a case study in transcendental meditation (Schreiber et al, 2017), a spiritual development technique, which was popularized by former Hindu ascetic Mharishi Mahesh Yogi and gained popularity in the west during the 1960's (Holzel et al, 2010). The concurrent brain states associated with transcendental meditation have been viewed as something outside of the world of physical measurement and objective evaluation by most scientific communities. Scientists now have the ability to measure and register electric potential of the human brain through the use of electroencephalographic technologies. One approach is to study finite differences within the minds of those practicing meditation, and those who do not. Such an endeavor is an avenue towards modeling a wide range of brain states (Lin, 2010). The combination of electroencephalographic data with modeling methods in fields such as data mining and bioinformatics could be used to prove that subjects in a state of transcendental meditation are in a verifiable and observable state of mind that can be monitored and predicted (Schreiber et al, 2017). Experiments found that cancer patients that practiced meditation experienced higher well-being levels, better cognitive function and lower levels of inflammation than a control group (Oh et al, 2009).

Therefore, a platform for comprehensive EEG data storage and processing is desirable to promoting applications of using EEG tools in both physiological (e.g., clinical uses, sleep evaluation, fatigue detection, etc.) and psychological (cognitive sciences, BCI, etc.) scopes. Such a platform consists of EEG data collection devices (viz., EEG headset), communication channels (e.g., smart phones), a web server that provides a web interface for users to access stored EEG data and activate data analysis algorithms, and an online database for EEG data storage and processing (Subedi et $\mathrm{al}, 2016)$. The system provides functionalities in data transition and storage, data visualization, and real-time data analysis via a web user interface. Experimental results obtained by applying various machine learning algorithms, including k-nearest neighbor, linear regression, and support vector machine, to accumulated EEG data sets in the database returned promising results with correct recognition rates as high as $90 \%$.

\section{EXISTING WORKS}

A cursory look into the topic revealed a wealth of information, much theoretical and limited to large government organizations and research facilities with huge budgets. For instance, the government has a program called the "Brain Research through Advancing Innovative Neurotechnologies ${ }^{\mathrm{TM}}$ (BRAIN)". The web site states the following: "The Brain Research through Advancing Innovative Neurotechnologies ${ }^{\mathrm{TM}}$ (BRAIN) Initiative is part of a new Presidential focus aimed at revolutionizing our understanding of the human brain. By accelerating the development and application of innovative technologies, researchers will be able to produce a revolutionary new dynamic picture of the brain that, for the first time, shows how individual cells and complex neural circuits interact in both time and space. Long desired by researchers seeking new ways to treat, cure, and even prevent brain disorders, this picture will fill major gaps in our current knowledge and provide unprecedented opportunities for exploring exactly how the brain enables the human body to record, process, utilize, store, and 
retrieve vast quantities of information, all at the speed of thought". The site even contains funding opportunities for companies and research facilities to participate and contribute to the program. Examples such as this can be found in abundance and what quickly becomes apparent is that there is a thirst for more knowledge about the human brain and how it works.

Very little information exists in the hobby and home space for EEG devices. Organizations such as OpenEEG and OpenBCI are available and facilitate the information sharing amongst hobbyists and attempt to inform the general public about the subject of gathering brainwave data. Companies like NeuroSky and Emotive sell headset EEG devices and provide software development kits (SDK) that include the tools necessary to gather brainwave data, but are limited to only reading brainwaves. In research perspectives, there is still space to gather more information, to have an enhanced data model, and see additional dependencies while the brain performs or reacts to specific tasks.

On the brain state modeling side, two types of research models have been used: statistical models and micro models. Statistics models are built by applying statistical analysis to collected data from meditation practitioners, while micro models try to catch physiological features of the brain state under examination. Current literatures show that both methods are used in the study of complementary and alternative medicine, which includes meditation as one of the methods. Loizzo et al (2010) performed a 20 -week contemplative self-healing program study, which showed that a contemplative self-healing program can be effective in significantly reducing distress and disability among the testers. Habermann et al (2009) on the other hand, performed a long-term (5-20 years) project to investigate the use of complementary and alternative medicine and its effects on the testers' health. Comparisons across different groups of people are also found. For example, in a 6-week mindfulness-based stress reduction program, subjects assigned to the program demonstrated significant improvements in psychological status and quality of life compared with usual care (Lengacher et al, 2009). Another comparison is found where a group of Qigong practitioners were compared to a control group and positive indicators were found in the study (Oh et al, 2008). A recent work that employs EEG data in brain disorder diagnosis is a systematic performance evaluation of the optimum path forest (OPF) classifier when coping with the task of epilepsy diagnosis directly through EEG signal analysis (Nunes et al, 2014), which reported $89.2 \%$ as average accuracy scores achieved by the OPF classifier when configured with Coiflets as feature extractors.

A survey of the literature on cognitive impairment and cancer presented in (Biegler et al, 2009) suggests that meditation may help improve cancer-related cognitive dysfunction and alleviate other cancer-related sequelae. It is well understood that although statistical studies can provide evidence for the effectiveness of meditation, it fails to provide a systematic view of human's epistemology and psychology. This addresses the needs for micro models that depict the inter-relationship between human's mind and physical body (Li et al, 2015a). To accurately and objectively record moods when one is practicing meditation, we seek a solution which could objectively measure the effectiveness of meditation in real time (Lin, 2010; Li et al, 2015b). Such work, when applied to real life, could help develop procedures that aim at healing certain applicable types of mental diseases with calibrating means to ensure efficacy.

In the following sections, we will describe a prototyping EEG headset we built for data collection, the online system for data storage and processing, and the web interface through which users can interact with the online database and perform dynamic data analysis and modeling. In the end, as a showcase of the usefulness of the dynamic modeling system, we present a simple dynamic model for a set of selected brain states, including meditation.

\section{A PROTOTYPING EEG HEADSET}

We briefly describe how a simple EEG headset can be built using open source materials. The prototype multi-functional headset we built consists of an EEG sensor, a pulse sensor, a temperature sensor, a microprocessor, and a microprocessor blue tooth shield. 
1. EEG sensor, commercial product from NeuroSky. The NeuroSky technology was chosen for its dry sensors capabilities. This means that the sensor requires no special liquid chemicals while making contact with the skin to read brainwaves.

2. Pulse Sensor, Open Source pulse sensor from pulsesensor.com. The pulse sensor is a current to voltage converter Op Amp circuit that uses a photodiode as current source. It has a Low Pass Filter for output.

3. Temperature sensor, commercial integrated circuit sensor, TMP36 - Analog Temperature sensor from Adafruit. The TMP36 temperature sensor is a solid state device. Meaning it does not use mercury. Instead, it uses the fact that as temperature increases, the voltage across a diode increases at a known rate. By precisely amplifying the voltage change, it is easy to generate an analog signal that is directly proportional to temperature.

4. Microprocessor: Arduino Mega 2560, Open Source.

5. Microprocessor Blue Tooth Shield: Bluetooth Low Energy (BLE) Shield from redbear.com. Added to the Arduino for low energy blue tooth communications with the iPhone.

The assembled headset is shown in Figure 1, where the 3 sensors are mounted on the tips of 3 legs in the forehead, the microprocessor and the microprocessor blue tooth shield are mounted on the back, and the ear lobe is used as the base of the EEG sensor.

Figure 1. EEG headset
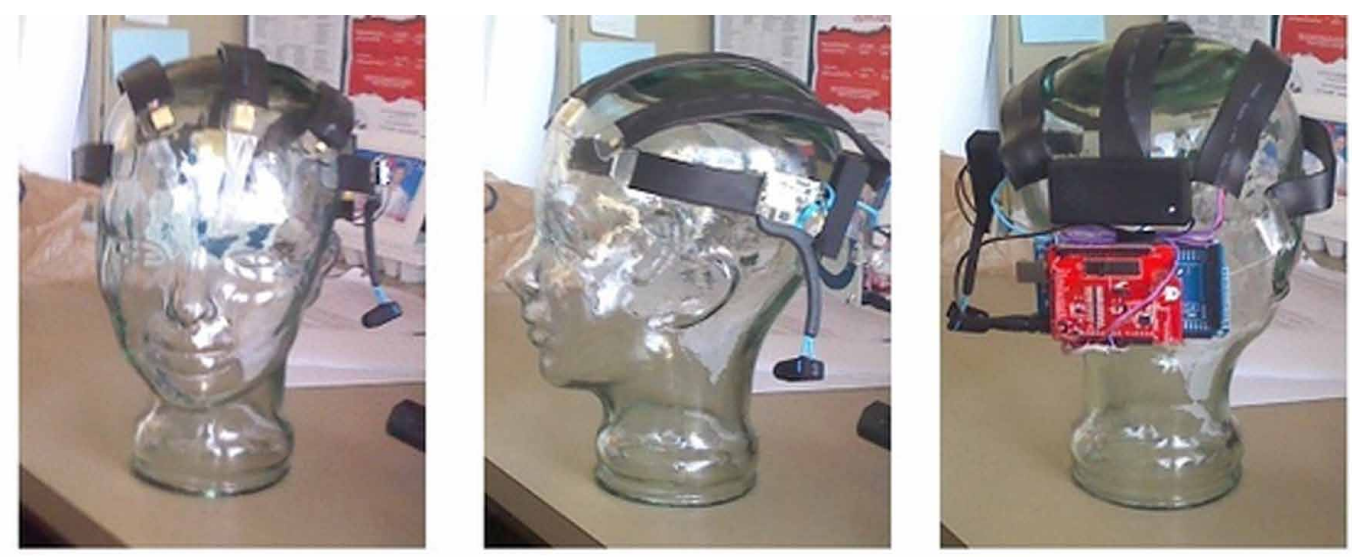

In order to test and validate that the headset is working properly and that all the sensors are functioning, a test environment had to be constructed. To simulate a real world environment, a mobile Smart Phone application was developed on the Apple iPhone platform. This platform was chosen for ease of access to development tools and availability of software development kits (SDK) from all the hardware and chipset vendors. Both NeuroSky and Red Bear Labs included sample applications that were then easily transferred to a custom application using a simple view to display all the sensor values.

To show that the headset sensors are working a custom mobile application was developed to view the results. Sample screenshots of the application with the actual results are displayed in Figure 2.

\section{THE SYSTEM FOR DYNAMIC BRAIN STATE MODELING}

The brain emits electrical signals that are caused by neurons firing in the brain. The patterns and frequencies of these electrical signals can be measured by placing a sensor on the scalp. For example, 
Figure 2. iOS sensor headset application
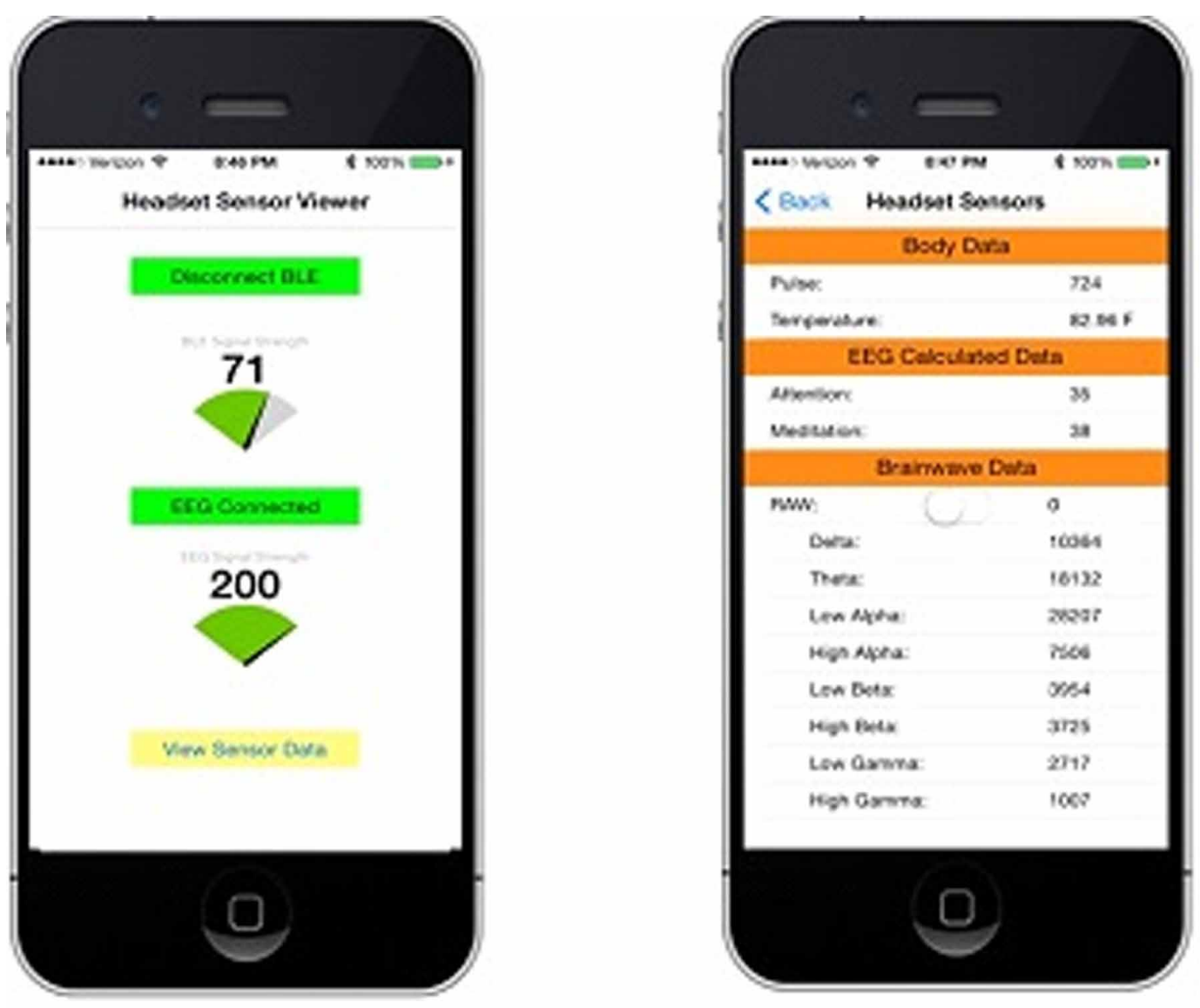

the EEG sensor by NeuroSky is able to measure the analog electrical signals commonly referred to as brainwaves and process them into digital signals to make the measurements available for further analysis. Table 1 lists the most commonly recognized frequencies that are generated by different types of brain activity.

Emotions play an essential role in many aspects of our daily lives, including decision making, perception, learning, rational thinking and actions. To detect the emotion of a person, the first approach is based on text, speech, facial expression and gesture. This approach, needless to say, is not reliable to detect emotion, especially when people want to conceal their feelings. Some emotions can occur without corresponding facial emotional expressions, emotional voice changes or body movements. On the contrary, such displays could be faked easily. Using multi-modality approach can overcome this shortcoming to limited extent.

The new approach is through affective computing, which employs EEG signals recorded when users perform some brain activities and apply analytical algorithms to EEG data to detect the emotion. This approach is based on the fact that brain activities have direct information about emotion and EEG signals can be measured at any moment and are not dependent on other activities of the user such as speaking or generating a facial expression. Different recognition techniques can be used in different situations to maximize recognition rates.

We are investigating an automatic EEG-based emotion recognition system that can record the EEG signals from users and measure their emotions. The EEG data are filtered to get separate frequency 
Table 1. Brainwave frequencies

\begin{tabular}{|l|l|l|}
\hline \multicolumn{1}{|c|}{ Brainwave Type } & \multicolumn{1}{|c|}{ Frequency Range } & \multicolumn{1}{c|}{ Mental States and Conditions } \\
\hline Delta & $0.1 \mathrm{~Hz}$ to $3 \mathrm{~Hz}$ & Deep, dreamless sleep, non-REM sleep, unconscious \\
\hline Theta & $4 \mathrm{~Hz}$ to $7 \mathrm{~Hz}$ & Intuitive, creative, recall, fantasy, imaginary, dream \\
\hline Alpha & $8 \mathrm{~Hz}$ to $12 \mathrm{~Hz}$ & Relaxed, but not drowsy, tranquil, conscious \\
\hline Low Beta & $12 \mathrm{~Hz}$ to $15 \mathrm{~Hz}$ & Formerly SMR, relaxed yet focused, integrated \\
\hline Midrange Beta & $16 \mathrm{~Hz}$ to $20 \mathrm{~Hz}$ & Thinking, aware of self and surroundings \\
\hline High Beta & $21 \mathrm{~Hz}$ to $30 \mathrm{~Hz}$ & Alertness, agitation \\
\hline
\end{tabular}

Table 2. Brain state recognition rates

\begin{tabular}{|l|l|l|l|l|}
\hline & \multicolumn{1}{|c|}{ SVM } & \multicolumn{1}{c|}{ kNN } & \multicolumn{1}{c|}{ Naïve Bayes } & \multicolumn{1}{c|}{ AdaBoost.M1 } \\
\hline Emotion Recognition Rate & $89.25 \%$ & $83.35 \%$ & $66 \%$ & $92.80 \%$ \\
\hline
\end{tabular}

Table 3. Recognition rates of AdaBoost.M1

\begin{tabular}{|l|l|l|l|l|l|}
\hline & \multicolumn{1}{|c|}{ Delta } & \multicolumn{1}{c|}{ Theta } & \multicolumn{1}{c|}{ Alpha } & \multicolumn{1}{c|}{ Betta } & All \\
\hline Emotion Recognition Rate & $69.95 \%$ & $68.40 \%$ & $75.50 \%$ & $89.70 \%$ & $92.80 \%$ \\
\hline
\end{tabular}

Figure 3. EEG data analysis flowchart

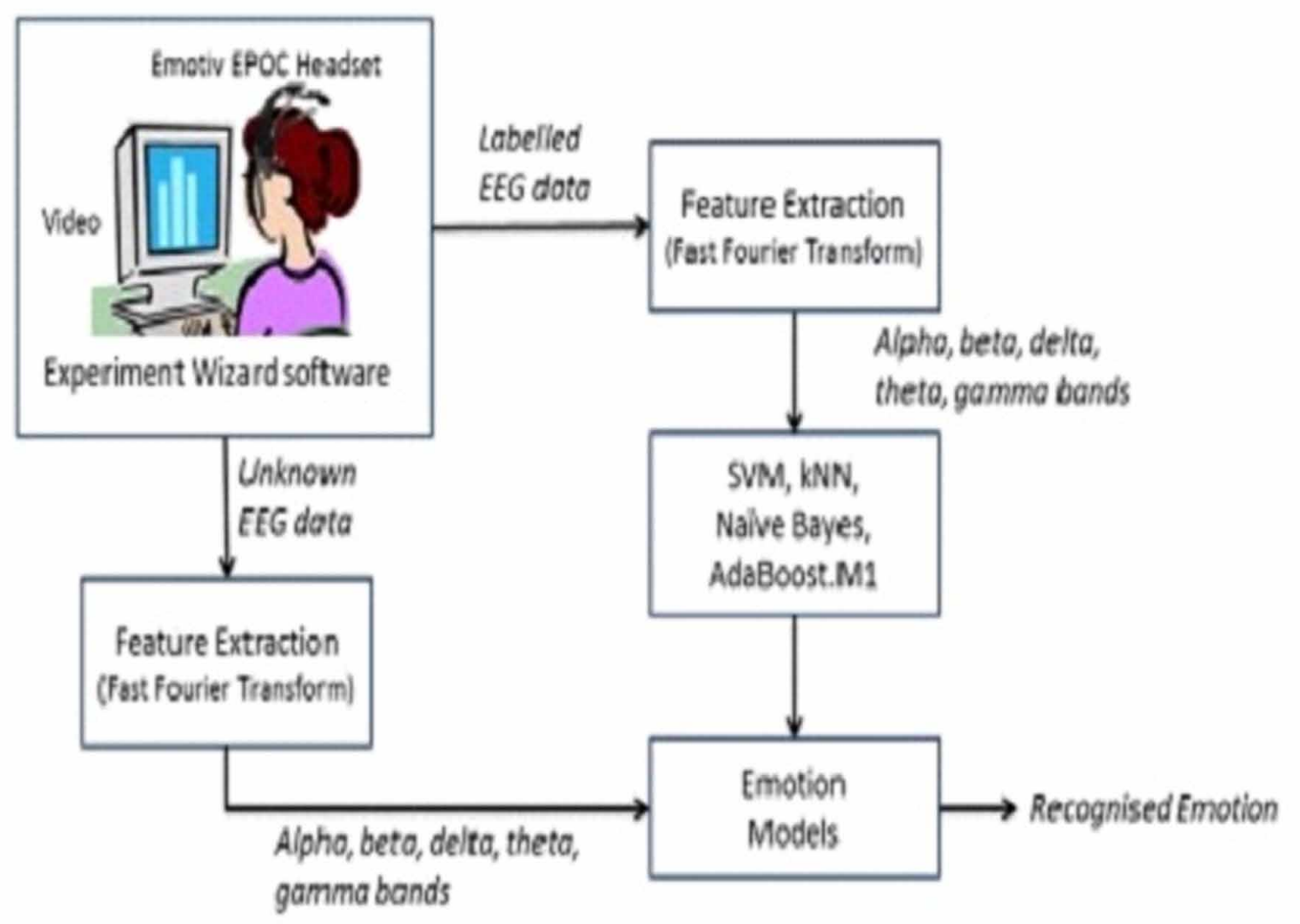




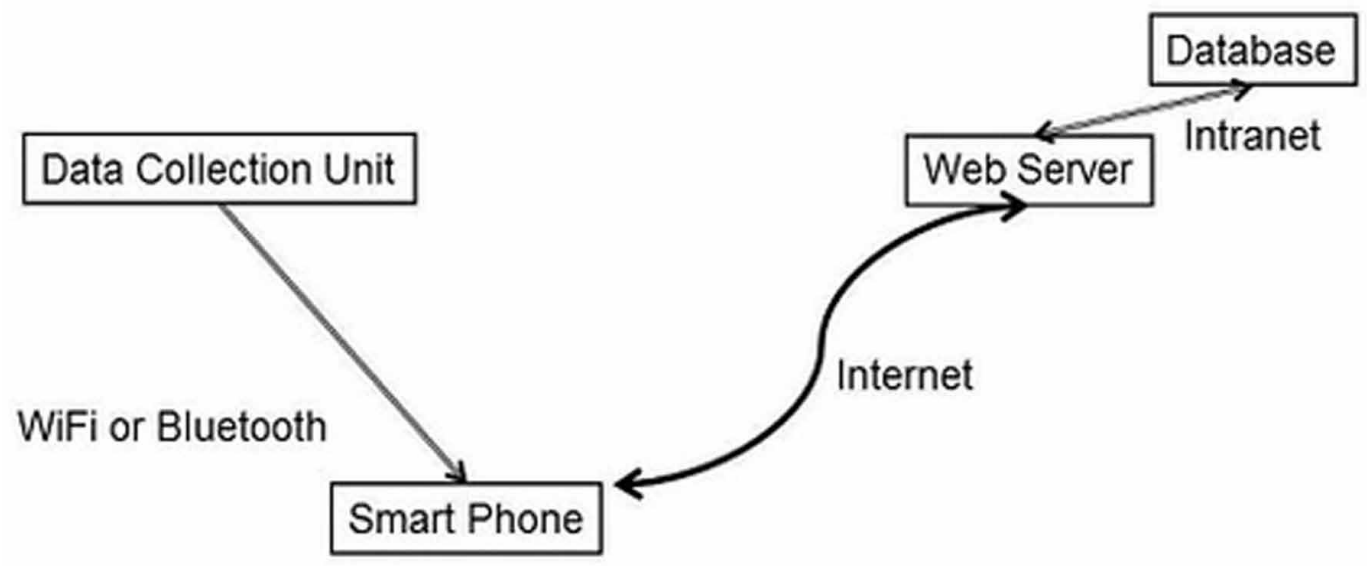

bands to train emotion classifiers with the four well-known classification techniques that are SVMs, Naïve Bayes, $k N N$ and AdaBoost.M1. Figure 3 shows the typical flowchart of data processing.

Table 2 shows the brain state recognition rate of different algorithms and Table 3 shows the band-wise recognition rate of the AdaBoost.M1 algorithm.

As depicted above, using EEG data, cognitive psychologists can visualize and observe correlations between different active brain states. It is desirable to create an application that takes EEG data and exposes it to various analytical techniques so the resultant brain states can be studied and predicted. We present the design and implementation of a system that integrate onsite EEG data collection, analysis, web based EEG data storage and modeling tools, and user feedback through mobile communication devices. Architecture of the system is shown in Figure 4.

The web server provides a user interface that allows users to view EEG data in the database and run R program to perform data analysis. Figure 5 shows that data are rendered in wave form mode and statistical mode, respectively. Figure 6 shows that $\mathrm{R}$ is invoked to perform data analysis tasks in interactive mode.

iPhone users can also use the web interface to connect to the database to view data. Figure 7 shows the two functions, viz., "collect data" and "view data", that a user can choose on the iPhone app (Figure 7(a)). The user can display data in text mode by viewing individual data frames (Figure 7(b)), or display the wave form of recorded data in certain time period (Figure 7(c)).

\section{DYNAMIC MODELING OF SAMPLE CASES}

We collected EEG data from 15 volunteer subjects using the Neurosky Mobile Headset. Three sets of data were collected in three brain states, viz., meditation, watching movie, and reading aloud. Data were then analyzed using Microsoft's spreadsheet software and statistical computing programming languages, $\mathrm{R}$ and Python. Component frequencies, including five major brain waves- Delta(1-3Hz), Theta(4-7Hz), Alpha Low(8-9Hz), Alpha High(10-12Hz), Beta Low(13-17Hz), Beta High(18$30 \mathrm{~Hz})$, Gamma Low $(31-40 \mathrm{~Hz})$, and Gamma Mid $(41-50 \mathrm{~Hz})$ were extracted from raw dataset. These frequencies represent specific brain states including deep meditation and high anxiety.

The data in the headset reports brain wave frequencies as a function of its power spectrum. Fourier Transform analysis was implemented by the application software package to decompose the raw EEG 
Figure 5. EEG data rendering on web interface

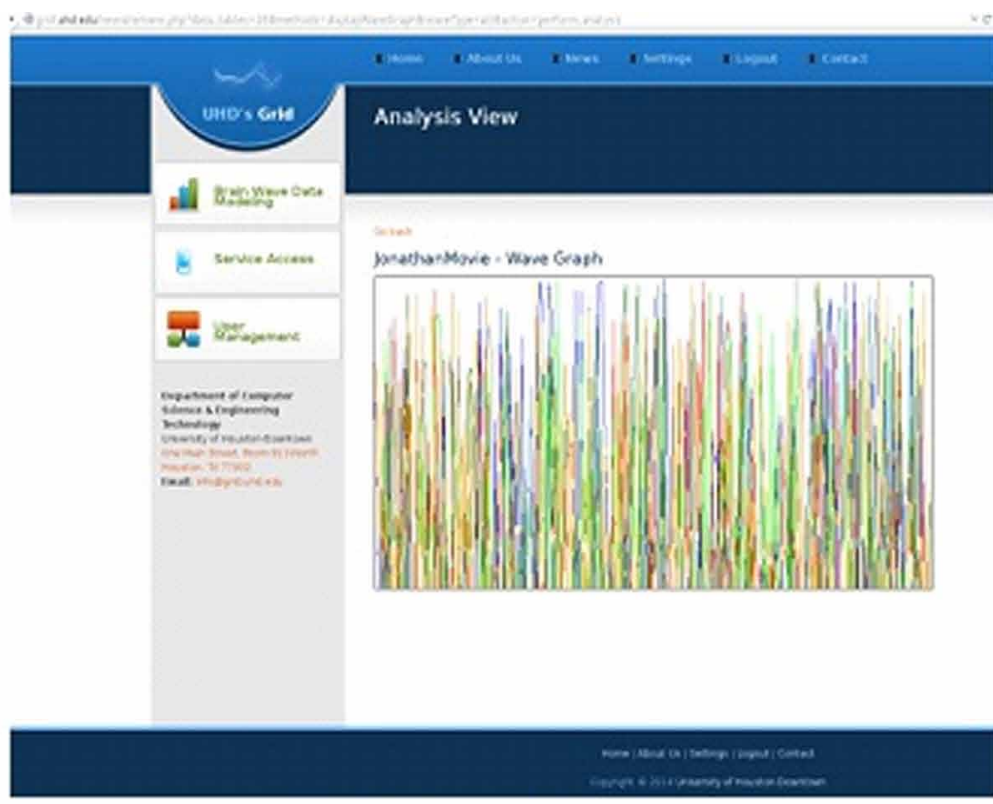

(a) EEG Wave forms

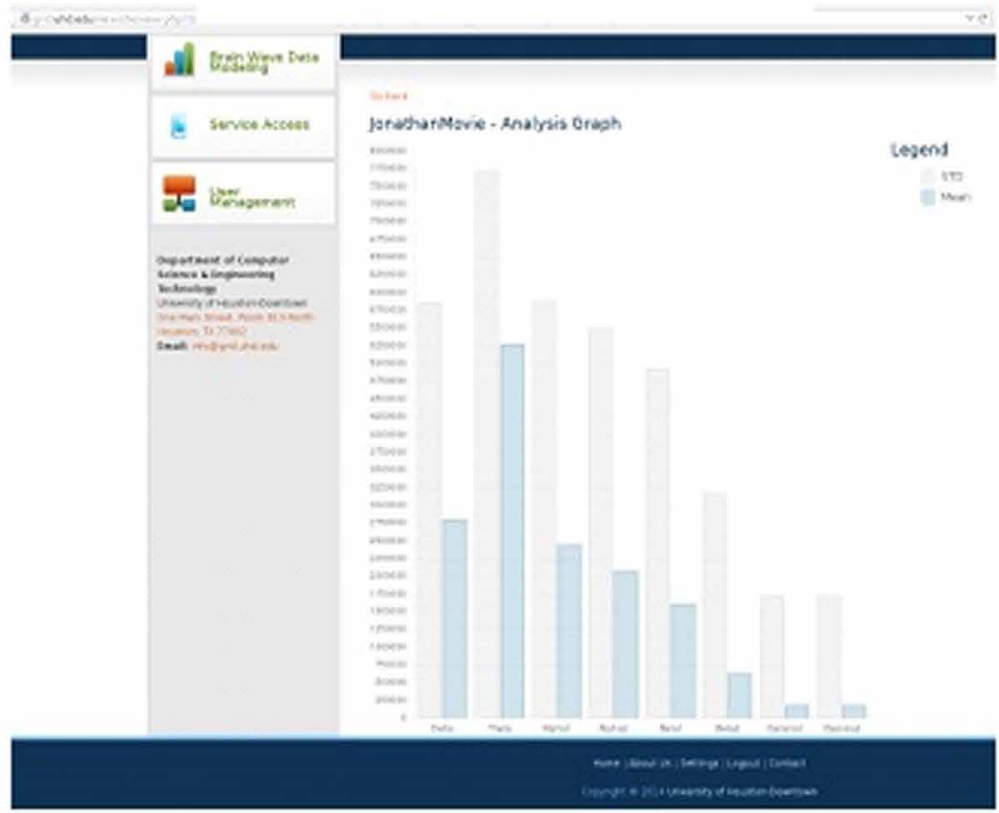

(b) EEG Histogram

time series into a voltage by frequency spectral graph (power spectrum). This power spectrum values obtained for specific brain waves was investigated for the numerical analysis of Quantitative EEG data.

The power spectrum data was normalized to reduce variability, which might have occurred due to difference in contact distance between the headset and the user, and changes in environmental 


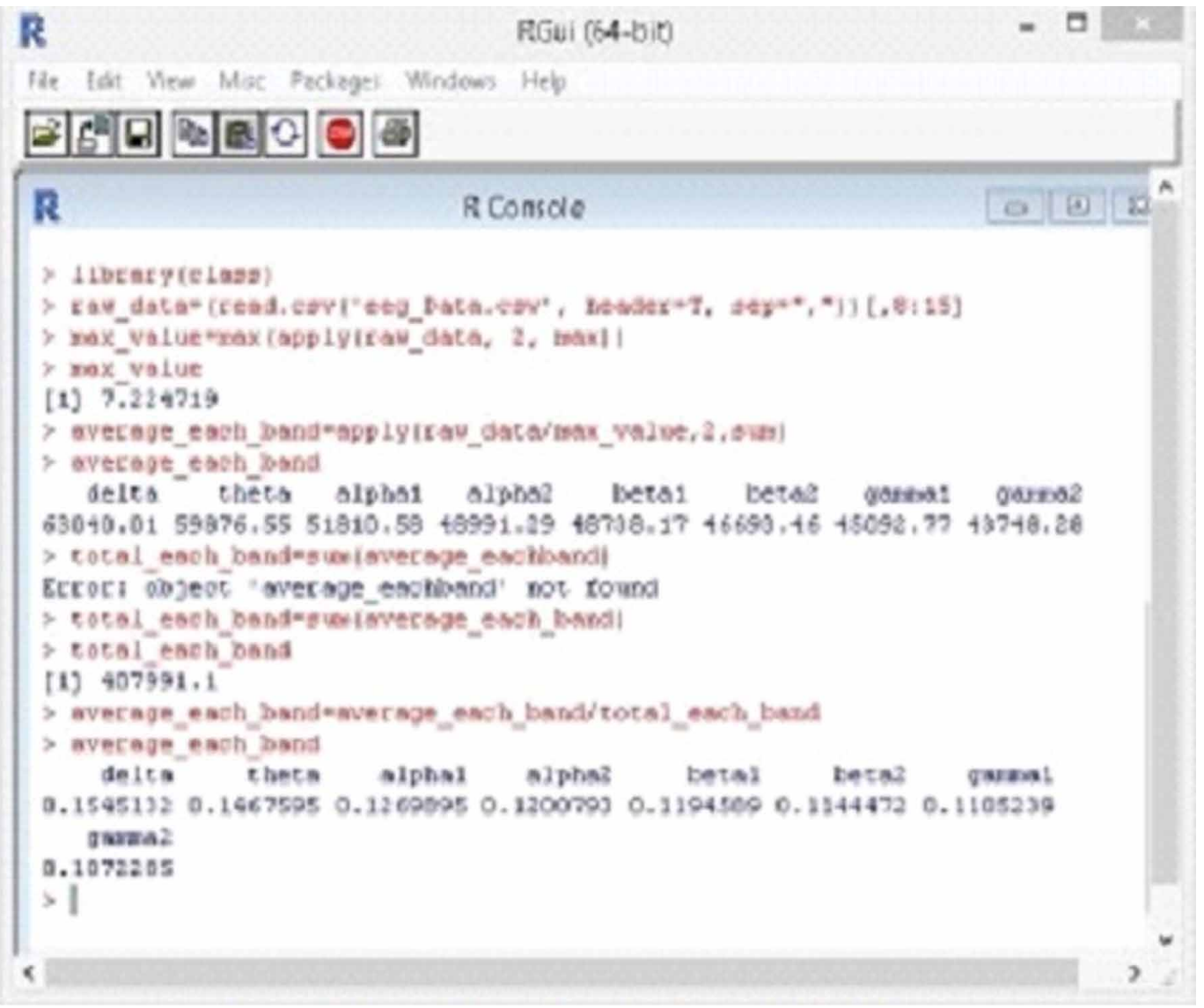

condition. In addition, automatic scaling feature in the hardware accounts for noises that make the data values large. The normalized method used the sum of all the eight brain waves power spectrum data and divided each data point by the sum to scale it within the range of 0 to 1 . The box plot (Figure 8) graphically depicts the numerical spread of the normalized data for the combined brain waves as well as meditation, movie watching, and reading aloud brain states. The standard deviation model was calculated for each brain state to investigate the variability of data and predict the percentage of values that are present within the one standard deviation from the mean (Figure 9).

The combined data set consisting of all the five major brain waves show analogous data variability, and presence of maximum outliers amongst alpha waves (Figure 8). Within three brain states, meditation showed the highest data spread. The deviation model correctly predicts that as the brain state changes from meditation to movie watching and reading, the higher frequency waves also changes from lower waves (delta and theta) to higher waves (alpha, beta, gamma). The result showed that the alpha waves are the most common waves among measured three different brain states (Figure 9 ). The recognition rates for deviation model of the three brain states using three classes are $64 \%$ for meditation, $65 \%$ for reading aloud, and $75 \%$ for watching a movie (Table 4 ). $64 \%$ in meditation is not a high level of acceptance, but it does make sense because none of the subjects are meditation practitioners. Mediation should be more discernible with samples of actual mediation practitioners.

Classification based advanced machine learning algorithm was implemented to further analyze the EEG data from different brain states. The main challenge in this process was the problem of data 


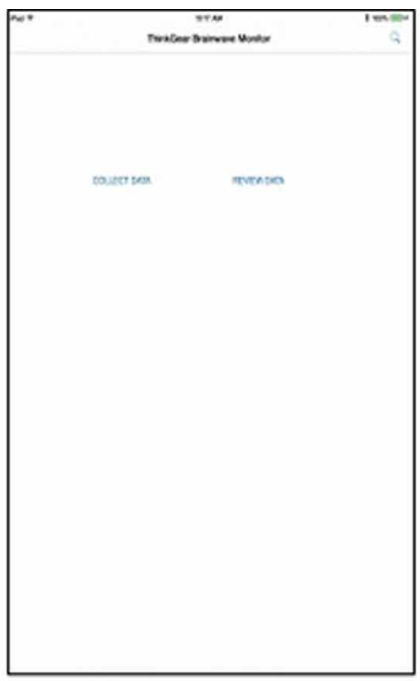

(a) Two Functions

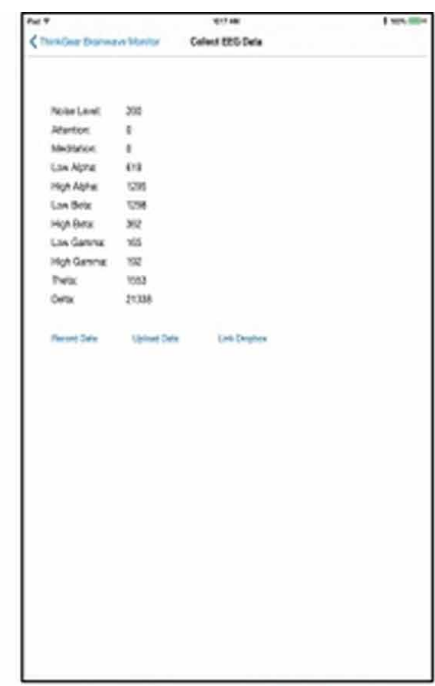

(b) Text mode display

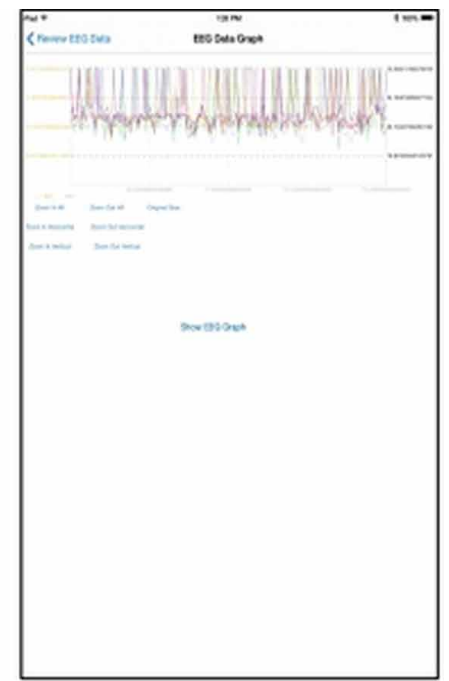

(c) Wave form

Fig. 7. iPhone Interface to the Web Server

separation for each brain wave at different brain states. Generally, the brain waves data from different states tend to cluster together, which becomes tedious for classification algorithms to draw a best fitting separation line.

The classification algorithms applied along with the obtained accuracy score is shown in Table 4. Due to the complexity of data clustering, as an initial step of modeling, two low frequency waves delta and theta brain waves were chosen as two variables and brain states- mediation, movie watching, and reading aloud were used as three nominal class values discretized as 0,1 , and 2 respectively. The results show that the K-Nearest model contributed the best prediction score with three classes - 78\% for mediation, $64 \%$ for reading aloud, and $71 \%$ for watching a movie. And for two classes - $89 \%$ for mediation combined with movie, and $85 \%$ for reading brain states.

Interestingly, when two class systems was used by combining meditation and movie watching as class 0 , and reading aloud as class 1 , the algorithm performed very well with lower error rate (Table 4). This finding highlights the fact that the majority of volunteers participated for EEG data collection are inexperienced meditator, and the data collected during movie watching and meditation are close to one another than reading aloud. Also, once again with two class system, K-Nearest model performed the best. Since, K-Nearest model depends on highest number of neighboring data point to classify itself to that particular group, the clustering effect of meditation and movie watching should have contributed to the superior performance of the K-Nearest algorithm (Figure 10).

Further, the dynamic fast Fourier transform analysis of EEG data was conducted to reveal the occurrence of dynamic frequency at a steady state along with the time series (Figure 11). The result shows comparison of FFL graph of three different brain states from two volunteers. In experiences meditator, the graphs show the localized energy of waves whereas in inexperienced meditator volunteer the localization of wave energy is low. This finding underscores the additional layer of complexity for analyzing EEG data. 
Figure 8. Visualization of normalized data sets using box plot (A) combined data, (B) meditation, (C) movie watching, and (D) reading aloud

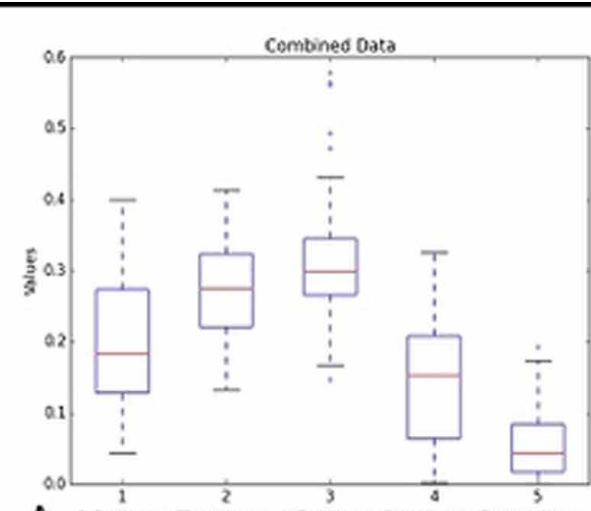

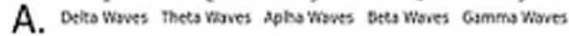

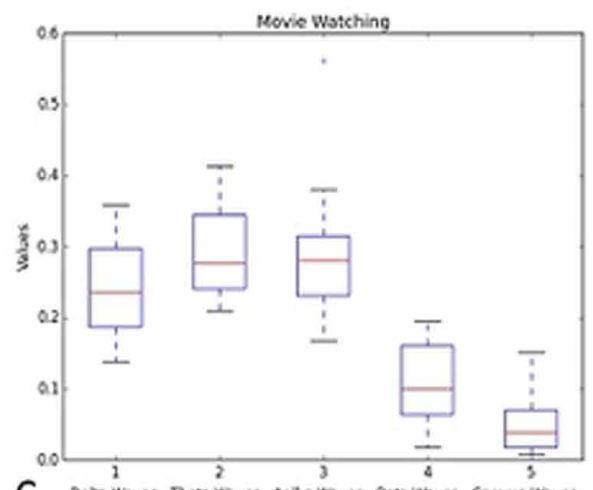

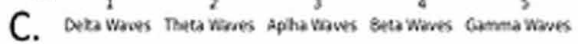

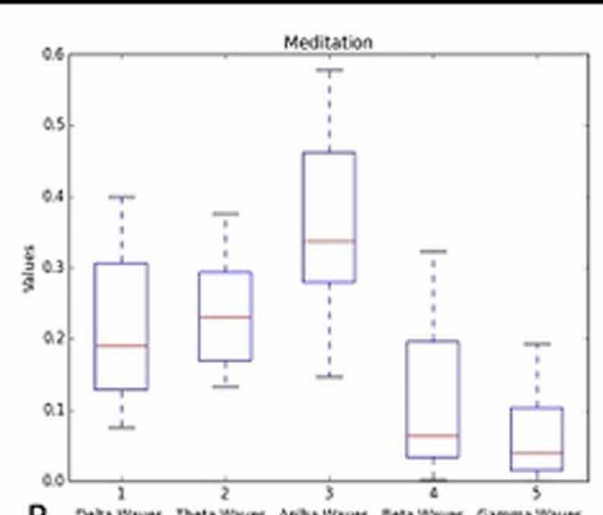

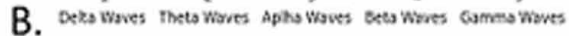

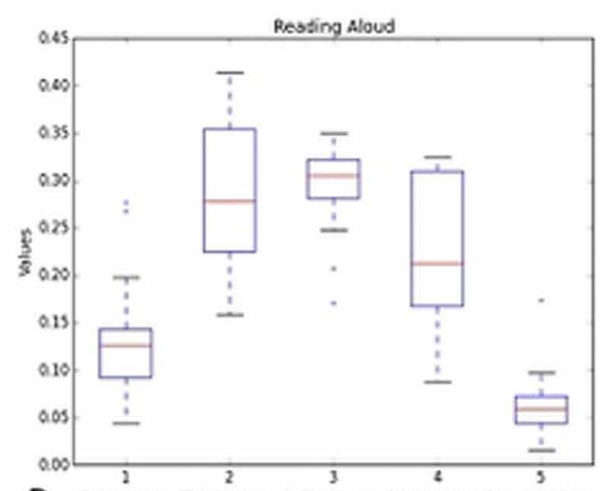

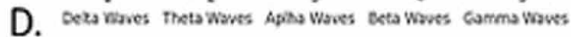

Figure 9. Standard deviation model for (A) Meditation, (B) Movie watching, and (C) Reading aloud brain states

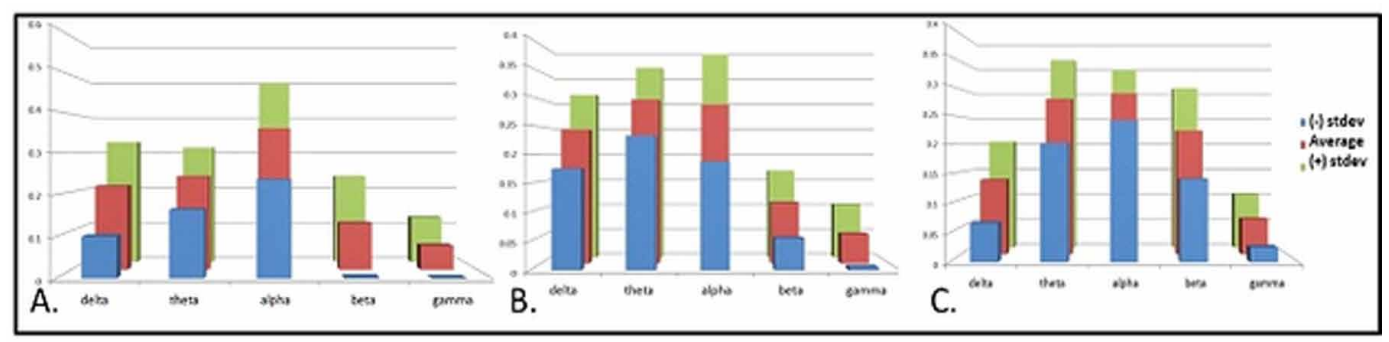

\section{FUTURE DIRECTIONS}

The challenge of modeling brain waves is to design an inclusive system, which incorporates all the major brain waves and addresses the variables such as specific regions of the brain, inconsistency within samples, limitations of the recording machine, and integrating knowledge form neurobiology in terms of understanding certain brain functions. There are center points in our brain, which generates 
Table 4. Summary of classification based prediction scores

\begin{tabular}{|c|c|c|c|c|c|c|c|}
\hline Model & Brain State & $\begin{array}{c}\text { Standard } \\
\text { Deviation } \\
\text { Model }\end{array}$ & $\begin{array}{c}\text { K Nearest } \\
\text { Neighbor }\end{array}$ & $\begin{array}{c}\text { Support } \\
\text { Vector } \\
\text { Machine }\end{array}$ & $\begin{array}{c}\text { Naïve } \\
\text { Bayes }\end{array}$ & $\begin{array}{c}\text { Logistic } \\
\text { Regression }\end{array}$ & $\begin{array}{c}\text { Multiclass } \\
\text { Algorithm }\end{array}$ \\
\hline $\begin{array}{c}\text { Three } \\
\text { classes }\end{array}$ & 0. Meditation & 64.29 & 78.57 & 78.57 & 50.00 & 78.57 & 50.00 \\
\hline & 1. Movie Watching & 75.71 & 71.43 & 57.14 & 71.43 & 21.43 & 35.71 \\
\hline & 2. Reading Aloud & 65.71 & 64.29 & 14.29 & 78.57 & 57.14 & 85.71 \\
\hline $\begin{array}{c}\text { Two } \\
\text { Classes }\end{array}$ & $\begin{array}{c}\text { 0. Meditation \& Movie } \\
\text { Watching }\end{array}$ & & 89.29 & 82.14 & & 85.71 & \\
\hline & 1. Reading Aloud & & 85.71 & 64.29 & & 78.57 & \\
\hline
\end{tabular}

different types of waves. For example, in Parkinson disease substantia-nigra region in the mid brain starts to dysfunction, which ultimately leads to lack of motor movement and coordination.

The method of separately analyzing the important known regions of the brain will provide better insight in the brain wave generation. Additionally, it imperative for brain wave modeling studies to contemplate the rigorous time series analysis of brain waves to decipher trend, irregularities, cycles, seasonality and other variations among waves during different states.

Technical difficulties with dry sensors (such as the one used by NeuroSky headset) includes low level of signal, artifacts, problems with hair, etc. limit their use, e.g., to control/communication. Most of the clinical EEG set is based on devices with up to 22 electrodes. More advanced devices with 128, 256, 512 electrodes are used for scientific purposes. This fact directs our study to move on to high quality EEG data with more advanced data collecting resources. The project, in its current stage, aims to address the complexity of classification of brain waves data by modeling the major brain waves independently with clinically significant brain regions combined with the time-series analysis. This will achieve an efficient and predictable brain wave modeling system which has potential application in hospitality and clinical industry for self-controlled deep brain relaxation and early diagnosis of various brain abnormalities respectively.

\section{CONCLUSION}

Brain state modeling using EEG data is a hard problem to tackle with given the uncertain nature of human's mind-body states. We present a method for building self-adjusting models using an online database system. The goal of the system is to provide users a tool for quick prototyping a brain state and the capability for dynamic adjusting the model according to the data currently available. This system consists of an EEG headset, applications on mobile phones, and a web server which provides an interface to the database and analytical tools.

We used meditation research to reach out to the high end applications of EEG data analysis in understanding human brain states and assisting in promoting human healthcare. The analysis of these states could be the initial step in a process to first predict and later allow individuals to control these states. We show-cased some initial dynamic models that can be distinguished from each other by running several classification algorithms on data sets we collected. While the state recognition rates vary across algorithms, the highest recognition rate we have reached is close to $90 \%$ by combining data from similar states. These results will seed more profound investigation on brain state modeling methods as well as EEG data collection procedures. 
Figure 10. Data analysis of brain waves. Standard deviation model for (A) Logistic Regression model three class (color: bluemeditation, orange-movie, and brown-reading), (B) Logistic Regression model two class (color: blue-meditation plus movie, and brown-reading), (C) K-Nearest model two class (color: pink-meditation plus movie, and blue-reading), and (D) Support Vector Machine with Linear Kernel two class (color: blue-meditation plus movie, and yellow-reading).

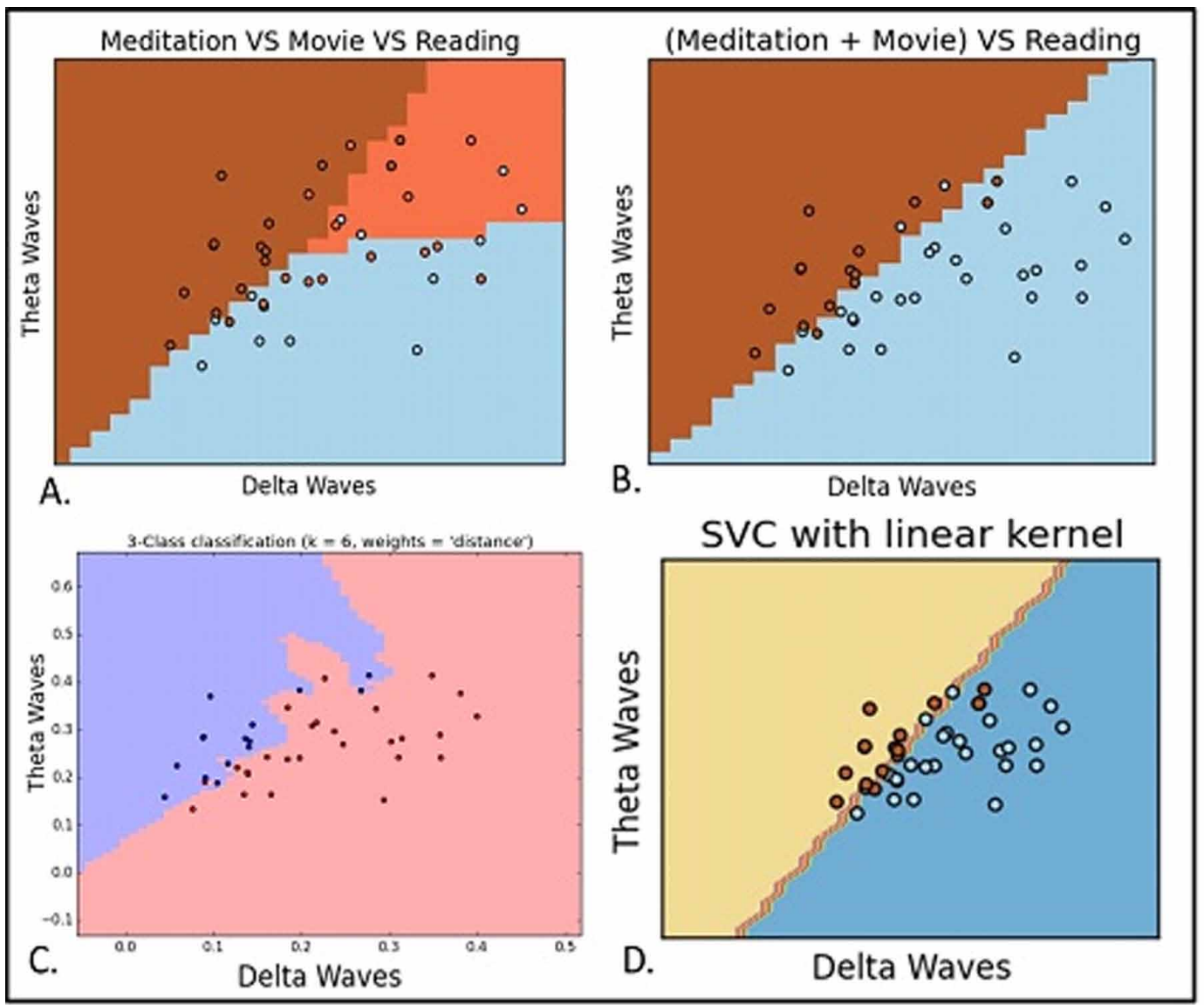

Fig. 10. Data analysis of brain waves. Standard deviation model for (A) Logistic Regression model three class (color: blue-meditation, orange-movie, and brownreading), (B) Logistic Regression model two class (color: blue-meditation plus movie, and brownreading), (C) K-Nearest model two class (color: pinkmeditation plus movie, and blue-reading), and (D) Support Vector Machine with Linear Kemel two class (color: blue-meditation plus movie, and yellowreading).

\section{ACKNOWLEDGMENT}

This work is partially supported by the National Computational Science Institute Blue Waters 
Figure 11. Dynamic Fourier Analysis for two volunteers (A-B-C: Inexperienced Meditator, D-E-F: Experienced Meditator) during meditation, movie watching, and reading aloud brain states

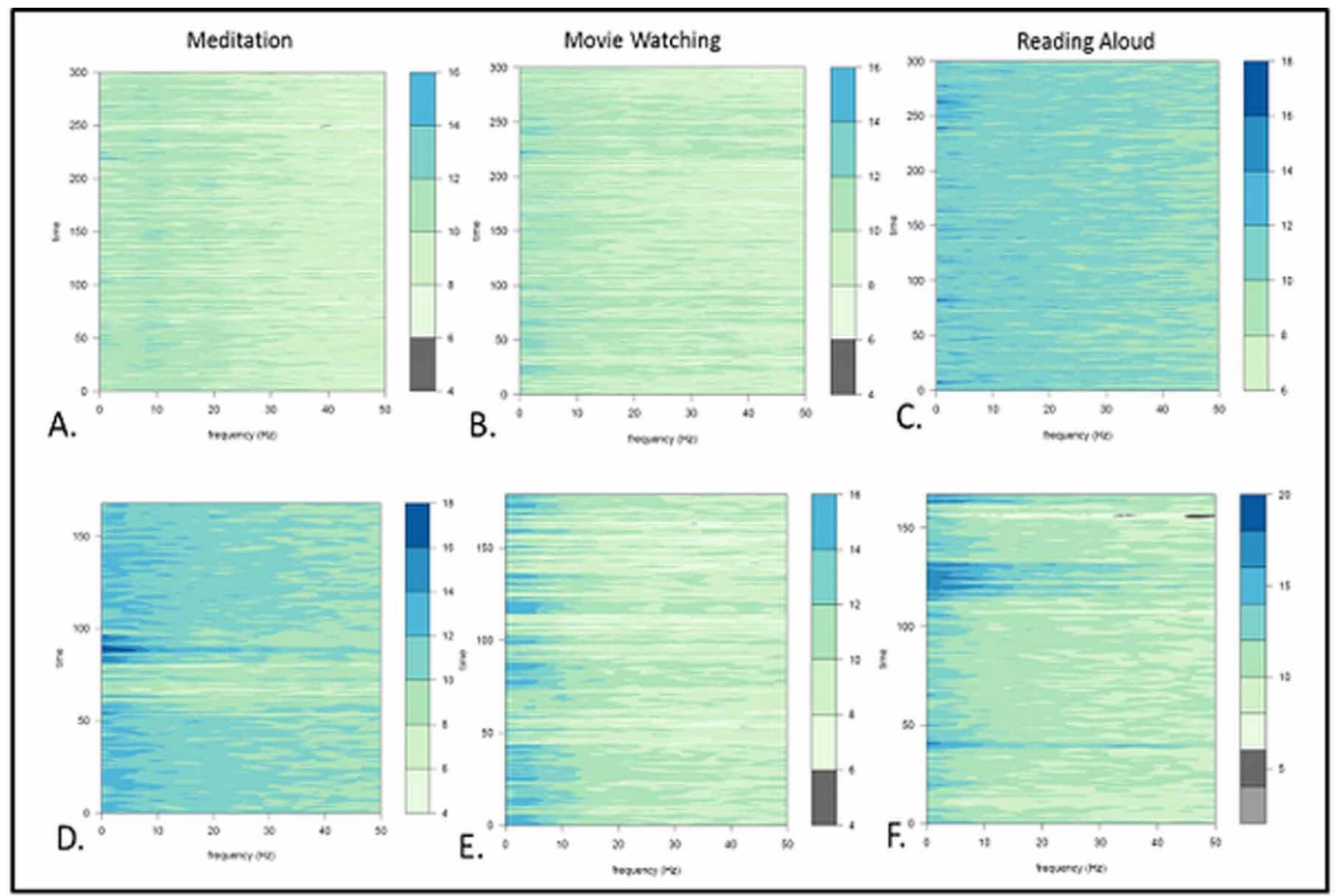

Fig. 11. Dynamic Fourier Analysis for two volunteers (A-B-C: Inexperienced Meditator, D-E-F: Experienced Meditator) during meditation, movie watching, and reading aloud brain states

Student Internship program, the Integrating High-Impact Practices at UHD grant from the Center for Teaching and Learning Excellence, and the Service Learning project from the Center for Community Engagement and Service Learning, the University of Houston-Downtown. 


\section{REFERENCES}

Biegler, K. A., Chaoul, M. A., \& Cohen, L. (2009). Cancer, cognitive impairment, and meditation. Acta Oncologica (Stockholm, Sweden), 48(1), 18-26.

Dressler, O., Schneider, G., Stockmanns, G., \& Kochs, E. F. (2004). Awareness and the EEG power spectrum: Analysis of frequencies. British Journal of Anaesthesia, 93(6), 806-809. PubMed

Freeman, W. J. (2000). A neurobiological interpretation of semiotics: Meaning, representation, and information. Information Sciences, 124(1-4), 93-102. doi:10.1016/S0020-0255(99)00144-9

Habermann, T. M., Thompson, C. A., LaPlant, B. R., Bauer, B. A., Janney, C. A., Clark, M. M., Rummans, T. A., Maurer, M. J., Sloan, J. A., Geyer, S. M., \& Cerhan, J. R. (2009). Complementary and alternative medicine use among long-term lymphoma survivors: A pilot study. American Journal of Hematology, 84(12), $795-798$. doi:10.1002/ajh.21554 PMID:19894247

Holzel, B.K., Carmody, J., \& Vangel, M. (2010). Mindfulness practice leads to increases in regional brain gray matter density. Psychiatry Research: Neuroimaging, 191(1), 36-43.

Lengacher, C. A., Johnson-Mallard, V., Post-White, J., Moscoso, M. S., Jacobsen, P. B., Klein, T. W., Widen, R. H., Fitzgerald, S. G., Shelton, M. M., Barta, M., Goodman, M., Cox, C. E., \& Kip, K. E. (2009). Randomized controlled trial of mindfulness-based stress reduction (MBSR) for survivors of breast cancer. Psychology (Irvine, Calif.), 18(12), 1261-1272. PMID:19235193

Li, Y., Chang, Y., \& Lin, H. (2015a). Cultivating Chan with Calibration. International Journal of Reliable and Quality E-Healthcare, 4(4), 32-51. doi:10.4018/IJRQEH.2015100102

Li, Y., Chang, Y., \& Lin, H. (2015b). Statistical Machine Learning in Brain State Classification Using EEG Data. Open Journal of Big Data, 1(2), 19-33.

Lin, H. (2010). Measurable Meditation. Proceedings of the International Symposium on Science 2.0 and Expansion of Science (S2ES 2010), the 14th World Multiconference on Systemics, Cybernetics and Informatics (WMSCI 2010), 56-61.

Lin, Y.-P., Wang, C.-H., Jung, T.-P., Wu, T.-L., Jeng, S.-K., Duann, J.-R., \& Chen, J.-H. (2010). EEG-Based Emotion Recognition in Music Listening. IEEE Transactions on Biomedical Engineering, 57(7), 1798-1806. doi:10.1109/TBME.2010.2048568 PMID:20442037

Loizzo, J. J., Peterson, J. C., Charlson, M. E., Wolf, E. J., Altemus, M., Briggs, W. M., Vahdat, L. T., \& Caputo, T. A. (2010). The effect of a contemplative self-healing program on quality of life in women with breast and gynecologic cancers. Alternative Therapies in Health and Medicine, 16(3), 30-37. PMID:20486622

Nunes, T. M., Coelho, A. L. V., Lima, C. A. M., Papa, J. P., \& de Albuquerque, V. H. C. (2014, July). EEG signal classification for epilepsy diagnosis via optimum path forest - A systematic assessment. Neurocomputing, 136(20), 103-123. doi:10.1016/j.neucom.2014.01.020

Oh, B., Butow, P., Mullan, B., \& Clarke, S. (2008). Medical Qigong for cancer patients: Pilot study of impact on quality of life, side effects of treatment and inflammation. The American Journal of Chinese Medicine, 36(3), 459-472. doi:10.1142/S0192415X08005904 PMID:18543381

Oh, B., Butow, P., Mullan, B., Clarke, S., Beale, P., Pavlakis, N., Kothe, E., Lam, L., \& Rosenthal, D. (2009). Impact of medical Qigong on quality of life, fatigue, mood and inflammation in cancer patients: A randomized controlled trial. Annals of Oncology : Official Journal of the European Society for Medical Oncology, 21(3), 608-614. doi:10.1093/annonc/mdp479 PMID:19880433

Schreiber, G., Lin, H., Garza, J., Zhang, Y., \& Yang, M. (2017). EEG visualization and analysis techniques. In Y. Zhang (Ed.), Health Informatics Data Analysis: Methods and Examples, Springer book series Health Information Science (pp. 155-168). doi:10.1007/978-3-319-44981-4_10

Subedi, S., Li, Y., Early, C., Chan, A., Garza, J., Schreiber, G., Chang, Y., \& Lin, H. (2016, March). A System for the Analysis of EEG Data and Brain State Modeling. In Emerging Trends in Computational Biology, Bioinformatics, and Systems Biology - Systems \& Applications. Elsevier/MK. doi:10.1016/B978-0-12-8042038.00030-4 
Zhou, S.-M., Gan, J. Q., \& Sepulveda, F. (2008). Classifying mental tasks based on features of higher-order statistics from EEG signals in brain-computer interface. Information Sciences, 178(6), 1629-1640. doi:10.1016/j. ins.2007.11.012

Hong Lin received his PhD in Computer Science in 1997 at the University of Science and Technology of China. Before he joined the University of Houston-Downtown (UHD), he was a postdoctoral research associate at Purdue University; an assistant research officer at the National Research Council, Canada, and an engineer at Nokia, Inc. Dr. Lin is currently an associate professor with UHD. His research interests include affective computing, parallel/distributed computing, grid computing, multi-agent systems, and high-level computational models. He is a co-supervisor of the Grid Computing Lab at UHD. He is also a senior member of the Association for Computing Machinery (ACM).

Jonathan Garza joined UHD's ACM organization and has become the Webmaster officer for it. Shortly thereafter, he joined the Scholar's Academy and became involved in research. This semester he had the honor of speaking at the 14th Annual UHD Student Research Conference about his group's research. He also presented the research done by the research group at the 1st Undergraduate Research Congress at Dallas in April. His research paper has been accepted for publication at the 2015 International Conference on Health Informatics \& Medical Systems, to be held at Las Vegas in July. The Conference paper acceptance rate has been between $26 \%$ and $28 \%$ for that conference. Finally, he was chosen to become a Peer Leader for Dr. Parker's class where he guided a group of students in their service-learning project.

Gregor Schreiber is a Senior IT Professional with 20 years of experience in IT Operations. Expertise includes large scale server hardware management in data centers, as well as monitoring, automation, and management. Special interests are Internet of Things (IOT), small scale microprocessors (Arduino), and sensor devices, including EEG, and mobile computing development. Presently working as a server analyst for Chevron Phillips Chemical Company. Graduated from UHD in 2014 with a Bachelor of Science in Computer Science. 